\title{
Prediction of 10-year Overall Survival in Patients with Operable Cervical Cancer using a Probabilistic Neural Network
}

\author{
Bogdan Obrzut ${ }^{1,2}$, Maciej Kusy³ ${ }^{3}$, Andrzej Semczuk ${ }^{\natural}{ }^{\bowtie}$, Marzanna Obrzut ${ }^{1}$, Jacek Kluska ${ }^{3}$ \\ 1. Medical Faculty, University of Rzeszow, Rejtana str. 16C, 35-959 Rzeszow, Poland \\ 2. Department of Obstetrics and Gynecology, Provincial Clinical Hospital No. 2, Lwowska str. 60, 35-301 Rzeszow, Poland \\ 3. Faculty of Electrical and Computer Engineering, Rzeszow University of Technology, Powstanców Warszawy avenue 12, 35-959 Rzeszow, Poland \\ 4. IIND Department of Gynecology, Lublin Medical University, Jaczewski str. 8, 20-954, Lublin, Poland \\ $\square$ Corresponding author: Professor Andrzej Semczuk, IIND Department of Gynecology, Lublin Medical University, Jaczewski str. 8, 20-954, Lublin, Poland; \\ e-mail address: andrzej.semczuk@umlub.pl
}

(c) The author(s). This is an open access article distributed under the terms of the Creative Commons Attribution License (https://creativecommons.org/licenses/by/4.0/). See http://ivyspring.com/terms for full terms and conditions.

Received: 2019.02.10; Accepted: 2019.06.02; Published: 2019.07.10

\begin{abstract}
Background: Toward the goal of predicting individual long-term cancer survival to guide treatment decisions, this study evaluated the ability of a probabilistic neural network (PNN), an established model used for decision-making in research and clinical settings, to predict the 10-year overall survival in patients with cervical cancer who underwent primary surgical treatment.

Patients and Method: The input dataset was derived from 102 patients with cervical cancer FIGO stage IA2-IIB treated by radical hysterectomy. We identified 4 demographic parameters, 13 tumor-related parameters, and 6 selected perioperative variables for each patient and performed computer simulations with DTREG software. The predictive ability of the model was determined on the basis of its error, sensitivity, and specificity, as well as area under the receiver operating characteristic curve. The results of the PNN predictive model were compared with those of logistic regression analysis and a single decision tree as reference models.

Results: The PNN model had very high predictive ability, with a sensitivity of 0.949 , a specificity of 0.679 , and an error rate of $12.5 \%$. The PNN's area under the receiver operating characteristic curve was high, 0.809 , a value greater than those for both logistic regression analysis and the single decision tree.

Conclusion: The PNN model effectively and reliably predicted 10-year overall survival in women with operable cervical cancer, and may therefore serve as a tool for decision-making process in cancer treatment.
\end{abstract}

Key words: cervical cancer; 10-year overall survival; probabilistic neural network; survival prediction

\section{Introduction}

Cervical cancer is the fourth most common malignancy among women worldwide [1]. In Poland, cervical cancer accounted for $3.6 \%$ of all newly registered carcinomas in women in 2015, making it the seventh most frequent female genital tract malignancy. Moreover, cervical carcinoma is the eighth leading cause of cancer-related death among Polish women [2].

Due to improved living conditions, growing health awareness, and the development of diagnostic, screening, and therapeutic methods over the last several decades, as well as the increasing life expectancy of the general population, cancer patients may also be surviving longer than before. Longer patient survival and delayed cancer deaths have been clearly confirmed in recently published data on many hematologic malignancies and solid tumors in humans, including cervical cancer [3-5]. Given these observations, long-term survival rates $(10,15$, or even 20 years) are becoming increasingly important 
outcome measures of cancer and are routinely reported by cancer registries worldwide $[3,6,7]$. The established estimates of long-term survival are useful for both clinical-practice and public-health purposes. They also enable estimation of general outcomes. They cannot, however, predict survival in an individual patient, a capability that might be very useful for planning individual follow-up.

A probabilistic neural network (PNN) - an advanced computer program that can analyze datasets and uncover complex relationships within them undetectable through logistic regression (LR) analysis - has been used to predict the 5-year overall survival of cervical cancer patients [8]. To our knowledge, however, there are no tools currently available for predicting 10-year survival in cervical cancer patients.

To achieve the goal of individualized prediction, in this study we sought to develop a universal PNN model for predicting 10-year overall survival in individual women with cervical cancer utilizing demographic characteristics, tumor-related parameters, and select perioperative data.

\section{Patients and Methods}

The study was approved by the Bioethics Committee of the Regional Medical Chamber, Rzeszow, Poland (reg. no. 3/98; 20/02/1998). Written informed consent was obtained from all the patients for their data to be used for investigation.

The study enrolled 117 women with cervical cancer classified as International Federation of Gynecology and Obstetrics (FIGO) stage IA2-IIB, who were operated on between 1998 and 2001 at the Department of Obstetrics and Gynecology of the Rzeszow State Hospital in Poland.

All of the patients underwent radical abdominal hysterectomy Piver class III and pelvic lymphadenectomy. Perioperative complications were prospectively recorded according to the classification proposed by Chassagne et al. [9].

After the postoperative recovery period, some patients received adjuvant radiotherapy. The inclusion criteria were as follows: lymph node metastases, lymph-vascular space invasion (LVSI), neoplastic tissue within the surgical incision, or non-squamous types of cervical cancer. Radiotherapy was administered as follows: teletherapy (50 Gy to the area of the pelvis minor in 25 fractions of 2 Gy; BOX technique) and brachytherapy (two fractions of low-dose rate; total dose, $30 \mathrm{~Gy}$ ). No patient from the study group received chemotherapy, since it was not routinely applied during the study period.

Follow-up was conducted every month during the first year after surgery, every 3 months during the second year, twice annually for 3-5 years, and then once per year. The 10-year follow-up data from all subjects were used to validate the examined computational intelligence models designed to predict death within 120 months.

Data available at the time of discharge, derived from histopathologic examination of the surgical specimens and data obtained during the follow-up were collected. In total, 23 variables were identified and sub-divided into 3 groups. The first group of variables comprised four demographic characteristics: age, body mass index (BMI), hormonal status, and the presence of concomitant diseases. The second group comprised 13 tumor-related parameters: FIGO stage, histologic type, histologic grade, tumor size $(\leq 4 \mathrm{~cm}$ or $>4 \mathrm{~cm}$ ), lymph node status, number of lymph nodes dissected, number of positive lymph nodes, lymph node ratio (ratio of positive to totally removed lymph nodes), lymph-vascular space invasion (LVSI), surgical-margin status, parametrial involvement, deep stromal invasion (outer one-third of the cervical stroma), and postoperative radiotherapy (Table 1). The third group included six selected perioperative variables: surgery time, blood loss, presence of intraoperative complications, presence of postoperative complications, types of complications, and length of hospital stay (Table 2).

All the above variables were used in computer simulations applying the PNN developed by Specht [10], which is available in DTREG software [11]. The error, sensitivity, specificity, and area under the receiver operating characteristic curve (AUROC) were used to determine the predictive ability of the applied model. The above parameters were obtained through a 10-fold cross-validation procedure [12]. The simulations were performed 20 times by assuming a random selection of training and test subsets. The results were averaged and the standard deviations calculated. As a reference model, we used LR analysis and a single decision tree (SDT), both of which are widely applied in medical research [13-17].

\section{Software}

PNN, SDT, and LR survival analyses were performed with DTREG software (version 10.7.18, Phillip H. Sherrod, www.dtreg.com, USA) and Matlab (version R2018b, MathWorks, Inc., www.mathworks .com, USA).

\section{Statistical analysis}

The AUROC values for the PNN and reference models were compared with two-tailed pairwise $t$-tests. A $p$ value of less than 0.05 was considered statistically significant. All statistical analyses were performed in MathWorks' Matlab R2018b software. 


\section{Results}

A total of 117 patients qualified for a radical Piver III hysterectomy and pelvic lymphadenectomy. Of these 117, 15 were excluded from the analysis (3 patients whose final histopathologic findings revealed endometrial cancer with cervical extension; 4 patients who continued postoperative treatment and follow-up at another institution; 3 patients who refused to participate in the study protocol; and 5 patients who were lost during the follow-up). The remaining 102 patients were considered eligible and were enrolled in the study.

Among the 102 included patients (mean patient age 48 years, range 29-73; mean \pm SD BMI $\left[\mathrm{kg} / \mathrm{m}^{2}\right]$ $25.9 \pm 4.9$ ), hormonal status was premenopausal in 71 and postmenopausal in 31. Concomitant diseases were found in 33: arterial hypertension in 21, diabetes mellitus in 3, ischemic heart disease in 6, and other diseases in 3 .

The prevailing histologic cervical tumor type was squamous-cell carcinoma (90\%). The clinicopathologic parameters are reported in Table 1.

Table 1. Clinicopathologic data of the study group.

\begin{tabular}{lll}
\hline Clinical stage n (\%) & IA2 & $15(15)$ \\
& IB1 & $51(50)$ \\
& IB2 & $8(8)$ \\
& IIA & $7(7)$ \\
Histologic type n (\%) & IIB & $21(20)$ \\
& Squamous & $91(90)$ \\
Histologic grade n (\%) & Non-squamous & $11(10)$ \\
& G1 & $19(19)$ \\
Tumor size n (\%) & G2 & $62(61)$ \\
& G3 & $21(20)$ \\
Mean number of removed lymph nodes (range) & $69(68)$ \\
Lymph node status n (\%) & Negative & $33(32)$ \\
& Positive & $13.8(1-40)$ \\
Mean number of positive lymph nodes & (range) & $25(76)$ \\
Lymph node ratio (range) & & $0.5(1-9)$ \\
LVSI n (\%) & Absent & $0.068(0-1)$ \\
& Present & $83(82)$ \\
Deep stromal invasion n (\%) & Absent & $19(18)$ \\
Parametrium infiltration n (\%) & Present & $66(65)$ \\
Surgical-margin status n (\%) & Absent & $36(35)$ \\
Postoperative radiotherapy n (\%) & Present & $78(77)$ \\
& Negative & $24(23)$ \\
& Positive & $89(88)$ \\
& Yes & $57(56)$ \\
& No & $45(44)$ \\
\hline
\end{tabular}

The perioperative parameters of the study group are shown in Table 2. Perioperative complications affected $46.1 \%$ of the patients. Most adverse events were mild or moderate complications that did not threaten patient health or life. Severe perioperative complications (pulmonary embolism, bleeding from the vena cava inferior, rupture of duodenal ulcer or genito-urinary fistulas) occurred in seven patients. Additional perioperative data are shown in Table 2.
The mean follow-up period was 95.5 months (range 6-120 months). During the follow-up, recurrence was identified in 28 patients $(27.5 \%)$. Pelvic recurrence was detected in 15 patients and the remaining 13 subjects were diagnosed with distant metastases. During the final observations, 74 patients were alive, and 28 had died from cancer-related causes. The overall 10 -year survival was $72.5 \%$.

The applied PNN model was used to predict the 10-year overall survival in cervical cancer patients treated with radical hysterectomy. The error, sensitivity, and specificity for the PNN were markedly better than those obtained by the LR model (Table 3). Although the SDT model had the highest sensitivity, its prediction error was higher than that of the PNN. The AUROC for the PNN was also substantially greater than that for both the LR and SDT models (Figure 1).

Of the 28 cases of patient death, 10 were incorrectly predicted to survive (Table 4). On the other hand, only four cases were misclassified among the patients who survived during follow-up.

Table 2. Perioperative parameters in the study group.

\begin{tabular}{lll}
\hline Mean surgery time (min, range) & & $194.7(80-310)$ \\
Mean blood lost ( $\triangle \mathrm{Hb}$; g\%; range) & & $3.8(0.3-7.8)$ \\
Intraoperative complications (n) & & 5 \\
$\begin{array}{l}\text { Postoperative complications (n) } \\
\text { Types of complications }\end{array}$ & Mild & 42 \\
according to Chassagne et al. [9] n (\%) & & $38(81)$ \\
& Moderate & $2(4)$ \\
& Severe & $7(15)$ \\
Mean hospital stay (days, range) & & $12.7(5-49)$ \\
\hline
\end{tabular}

Table 3. Error, sensitivity, specificity, and AUROC for PNN and the reference predicting models after a 10 -fold cross-validation procedure. The results are shown as means for 20 simulations, with standard deviations in parentheses.

\begin{tabular}{lllll}
\hline & Error & Sensitivity & Specificity & AUROC \\
\hline PNN & 0.125 & 0.949 & 0.679 & 0.809 \\
& $(0.024)$ & $(0.010)$ & $(0.064)$ & $(0.026)$ \\
LR & 0.300 & 0.816 & 0.393 & 0.622 \\
& $(0.008)$ & $(0.018)$ & $(0.032)$ & $(0.012)$ \\
SDT & 0.196 & 0.965 & 0.379 & 0.624 \\
& $(0.018)$ & $(0.011)$ & $(0.036)$ & $(0.013)$ \\
\hline
\end{tabular}

Table 4. Confusion matrix for the probabilistic neural network.

\begin{tabular}{lll}
\hline & \multicolumn{2}{l}{ Predicted outcome } \\
\hline Actual outcome & Died & Survived \\
Died & 18 & 10 \\
Survived & 4 & 70 \\
\hline
\end{tabular}

\section{Discussion}

Probabilistic neural networks are established tools for classifying medical data [18, 19], and were recently used for predicting complications as well as 5 -year overall survival in cervical cancer patients treated with radical hysterectomy $[8,20,21]$. A PNN 


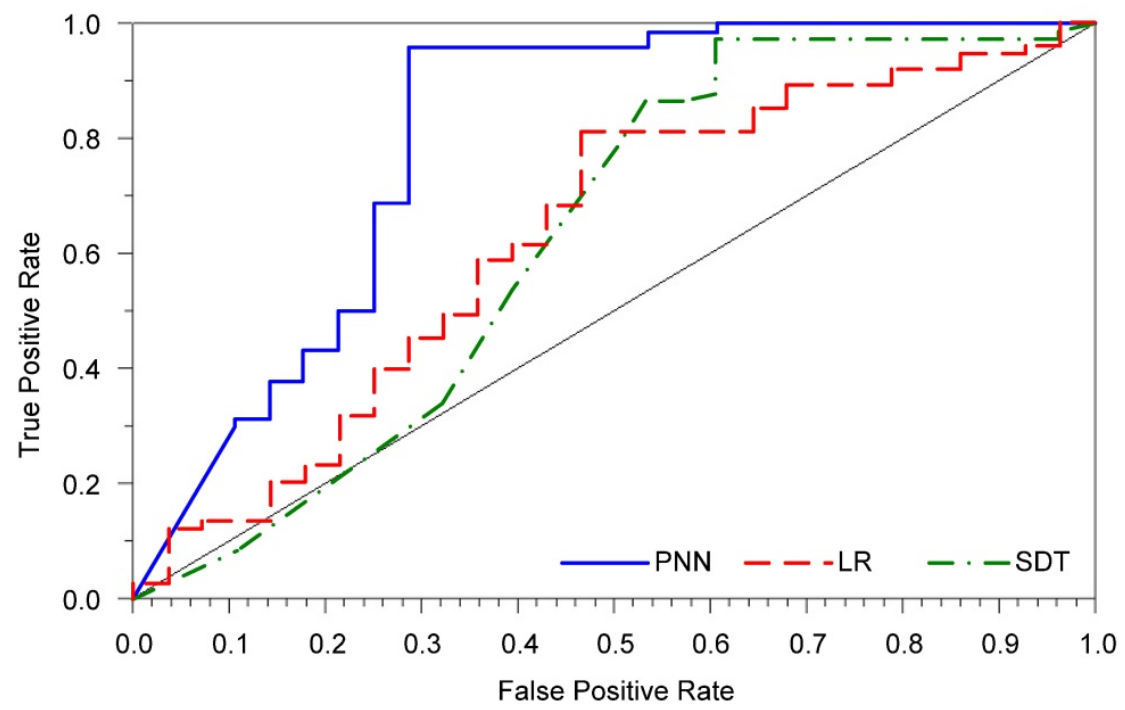

Figure 1. Receiver operating characteristic curves for all prediction models.

model for predicting long-term overall survival to aid in treatment decision-making, however, was lacking. A search of the PubMed ${ }^{\circledR}$ database revealed that there are no previously published reports of the application of a PNN to predict the effects of radical hysterectomy on 10-year overall survival in cervical cancer patients. This is also the first investigation of prognostication of 10-year survival in these patients.

Operationally, the most important advantage of PNN is that training is easy and instantaneous [10]. As additional patterns (e.g., records involving patient data) are observed and stored in the network, the generalization property of this network as the most important feature of each classification algorithm, will improve. Other advantages of the PNN are as follows: the shapes of the decision surfaces can be made as complex as necessary or as simple as desired by choosing the appropriate value of the smoothing parameter; the decision surfaces can approach the Bayes optimum; erroneous samples are tolerated; sparse samples are adequate for network performance; for time-varying statistics, old patterns can be overwritten with new patterns; and unlike many artificial neural networks, PNN operates completely in parallel.

Our simulations were performed using mostly prognostic factors that are well-established in uterine cervical carcinoma. The evidence for the included variables is discussed below. Clinical disease stage was included because it is a well-established critical variable [22, 23]. The FIGO stage was included because it was recently reported to be an independent prognostic factor for 10-year overall survival in women with cervical cancer [24, 25]. Although previous studies failed to confirm that tumor size is an independent prognostic factor for the 10-year survival rate [24, 25], we included tumor size because it correlates with outcome for patients with cervical carcinoma [26-28]. Additional established prognostic factors included in the study were parametrial infiltration and depth of stromal invasion of the cervix [25,29-31]. We also included histologic subtype, although no conclusive evidence regarding its effects on patient outcomes in cervical cancer has been presented [32]. Some studies found that 5-year survival rates do not differ significantly between squamous cell carcinoma and adenocarcinoma [33, 34], whereas others report that the 5-year survival rates are unfavorable in women with cervical adenocarcinoma [35, 36]. In our previous study with long-term follow-up [24], we identified a tendency toward higher 10-year disease-free survival and 10-year overall survival rates for patients with squamous cell carcinoma than for patients with adenocarcinoma. In agreement, Suprasert et al. [25] reported that histologic type of cervical cancer other than squamous cell-carcinoma was an independent unfavorable prognostic factor during a 10-year follow-up.

Despite the controversy surrounding histologic grade as a prognostic factor for patients with cervical cancer, we included histologic grade in the present study. Several findings indicate an unfavorable 5-year outcome for patients with poorly differentiated squamous cell cervical carcinoma [37], but other studies have not confirmed these observations [38, 39]. We previously reported a tendency toward lower 10-year survival rates for patients with poorly (G3) differentiated cervical carcinoma compared with patients with G1 and G2 tumors [24].

Lymph node involvement, another parameter included in the present study, is closely related to 5 -year survival in cervical cancer patients [24, 40-43]. The most recently reported data indicate that 10-year disease-free and overall survival are significantly lower for patients with positive pelvic lymph nodes than for patients without lymph node metastases [24, 25].

LVSI is associated with an unfavorable outcome in cervical cancer patients. Although the correlation between LVSI and lymph node involvement is high, some researchers consider LVSI an independent factor for predicting 5-year survival [44, 45]. We recently reported in a long-term follow-up study that 10 -year 
overall survival rates are significantly higher in patients without LVSI than in those with LVSI [24]. Interestingly, in that study, we also found that the 10-year disease-free survival rates tended to be higher for patients without LVSI [24].

We included patient age, although the literature includes contradictory data regarding the effect of patient age on 5-year overall survival in patients with cervical cancer. Several studies report no differences in survival rates among different age groups [46, 47], whereas others suggest that younger patients have a significantly poorer prognosis $[48,49]$. In our previous long-term follow-up study, we found no association between patient age and 10-year overall survival [24].

A positive surgical margin was included in the present study because it is a well-established important risk factor for cervical cancer recurrence. The effect of positive surgical margins on either 5-year or 10-year overall survival in cervical cancer patients has not been supported by multivariate analyses [24, 37, 50].

Available data do not support a direct effect of surgery duration or length of hospitalization on patient survival. Nevertheless, we included various perioperative variables as related to both the course of the operation and convalescence, and thus may also relate to complications. Similarly, perioperative complications themselves do not affect the natural history of carcinoma, but the treatment of perioperative complications may delay administration of adjuvant therapy, consequently negatively affecting patient prognosis. Comorbidity as an indicator of general patient health, may also limit the administration of adjuvant therapy, which might worsen the prognosis [51].

In our PNN model, we applied a total of 23 variables (see above: demographic characteristics, tumor-related parameters, and select perioperative data) because a larger number of factors improves the accuracy of artificial neural networks [52], and even variables without a statistically significant effect may still have some effect on survival [53]. Our model could predict 10-year overall survival in cervical cancer patients with an error of $12.5 \%$. The probabilistic neural network had high sensitivity but relatively low specificity, possibly as a result of class imbalance, i.e., the study population had a very low number of deaths [8].

Unfortunately, we cannot compare our findings with results from other studies due to the small number of relevant publications. Only a few studies in the current literature predict survival of cervical cancer patients, and they reported only 5-year overall survival. For example, Ochi et al. [54] used artificial neural networks to predict the effects of radiotherapy on survival of cervical cancer patients. Polterauer et al. [53] developed a nomogram for patients with uterine cervical carcinoma, FIGO stages IB-IV, whereas Zhou et al. [55] established a nomogram predicting the effects of surgical treatment on survival in patients with stage IA-IIB cervical cancer. All three models predicted 5-year overall survival with an AUROC value of $0.71-0.778$. Our recently published results predicting 5-year overall survival in cervical cancer patients with FIGO stage IA2-IIB cancer using computational intelligence methods outperformed all the aforementioned studies (AUROC value for PNN model of 0.818 ) [8]. These findings suggest that the present study, based on similar methodology, provides satisfactory and credible findings.

This study has some limitations. First, clinical data from patients operated on between 1998 and 2001 were used for the simulations. Since then, the treatment standards for cervical cancer substantially changed. In this context, it may seem controversial that we included patients with FIGO stage IIB cervical cancer. The current recommendations suggest that cervical cancer FIGO stage IIB should be treated with definitive chemo-radiation [56-58]. The latest NCCN-Guidelines (version 1.2019), however, state that "in some countries, select cases of stage IIB may be treated with upfront radical hysterectomy or neoadjuvant chemotherapy followed by radical hysterectomy" [56]. Surgical treatment of cervical cancer FIGO stage IIB is common in China, Korea, and Japan [59-62]. The German Cancer Society and German Society of Gynaecology and Obstetrics also recently recommended that FIGO stage IIB cervical cancer can be treated by radical hysterectomy with adjuvant radiotherapy [63]. Poland is among those countries with a longstanding surgical tradition [24, 43]. We emphasize that in our series of radical hysterectomy, selected cases of FIGO stage IIB cervical cancer were qualified by early parametrial infiltration (proximal portion of the lateral parametria) and associated pathologies of the female genital tract, including uterine fibroids, adnexal tumors, or massive ventral hernia in a post-laparotomy scar, as well as comorbid psychiatric disorders precluding radiotherapy. The survival outcomes for patients in the present study were comparable to those previously reported $[25,59,64$, $65]$, thus indirectly confirming our decisions. Second, concurrent chemo-radiotherapy has been introduced on a large scale. In this context, our results refer only to a select group of patients. Finally, the number of patients was limited to allow us to analyze in detail the effects of histologic type on survival in patients with non-squamous cell carcinoma. This category also included patients with adenocarcinomas and 
glandular squamous, microcellular, and undifferentiated cervical carcinomas.

The current study has several strengths. The main strength is the study design. As previously mentioned, on the basis of a PubMed ${ }^{\circledR}$ database search, this is the first report applying PNNs for predicting 10-year overall survival in cervical cancer patients treated with radical hysterectomy. Moreover, the entire series of patients was treated in a single institution in which the same principles of diagnostic and surgical procedures were carefully matched. This aspect markedly enhances the strength of our study.

\section{Conclusion}

The PNN model effectively and reliably predicted 10-year overall survival in women with operable cervical cancer, and may therefore serve as a tool for decision-making processes in cancer treatment.

\section{Abbreviations}

AUROC: Area Under Receiver Operating Characteristic curve; BMI: Body Mass Index; FIGO: The International Federation of Gynecology and Obstetrics; LR: Logistic Regression; LVSI: Lymph-Vascular Space Invasion; PNN: Probabilistic Neural Network; SDT: Single Decision Tree.

\section{Acknowledgments}

This study was supported by the grant (Dz. St. 326/2019 to A.S.) from Lublin Medical University, Lublin, Poland.

\section{Author Contributions}

BO initiated and co-led the study design, collected the data, co-led the data analysis and interpretation, co-wrote the first draft of the paper, coordinated draft revisions, and co-wrote the final manuscript. MK co-led the study design, co-led the data analysis and interpretation, co-wrote the first draft of the paper, and co-wrote the final manuscript. AS contributed to the data analysis and interpretation, and draft revision. MO contributed to data collection and interpretation. JK contributed to the study conception and design, data analysis and interpretation, and draft revision. All authors read and approved the final version of the manuscript.

\section{Competing Interests}

The authors have declared that no competing interest exists.

\section{References}

1. Bray F, Ferlay J, Soerjomataram I, et al. Global cancer statistics 2018: GLOBOCAN estimates of incidence and mortality worldwide for 36 cancers in 185 countries. CA Cancer J Clin. 2018; 68: 394-424.
2. [Internet] Didkowska J, Wojciechowska U, Olasek P. Cancer in Poland in 2015. http://www.onkologia.org.pl

3. Lim GH, Wong CS, Chow KY, et al. Trends in long-term cancer survival in Singapore: 1968-2002. Ann Acad Med Singapore. 2009; 38: 99-105.

4. Allemani C, Weir HK, Carreira H, et al. CONCORD Working Group. Global surveillance of cancer survival 1995-2009: analysis of individual data for $25,676,887$ patients from 279 population-based registries in 67 countries (CONCORD-2). Lancet. 2015; 385: 977-1010.

5. Allemani C, Matsuda T, Di Carlo V, et al. CONCORD Working Group. Global surveillance of trends in cancer survival 2000-14 (CONCORD-3): analysis of individual records for 37513025 patients diagnosed with one of 18 cancers from 322 population-based registries in 71 countries. Lancet. 2018; 391: 1023-75.

6. Brenner $\mathrm{H}$, Hakulinen T. Long-term cancer patient survival achieved by the end of the 20th century: most up-to-date estimates from the nationwide Finnish cancer registry. Br J Cancer. 2001; 85: 367-71.

7. Sjöberg J, Halthur C, Kristinsson SY, et al. Progress in Hodgkin lymphoma: a population-based study on patients diagnosed in Sweden from 1973-2009. Blood. 2012; 119: 990-6.

8. Obrzut B, Kusy M, Semczuk A, et al. Prediction of 5-year overall survival in cervical cancer patients treated with radical hysterectomy using computational intelligence methods. BMC Cancer. 2017; 17: 840-8.

9. Chassagne D, Sismondi P, Horiot J, et al. A glossary for reporting complications of treatment in gynecological cancers. Radiother Oncol. 1993; 26: 195-202.

10. Specht DF. Probabilistic neural networks and the polynomial Adaline as complementary techniques for classification. Neural Netw IEEE Trans. 1990; 1: 111-21.

11. [Internet] Sherrod PH. DTREG Predictive Modelling Software. http://www.dtreg.com.

12. Kohavi R. A study of cross-validation and bootstrap for accuracy estimation and model selection. Proceedings of the $14^{\text {th }}$ International Joint Conference on Artificial Intelligence - vol. 2, IJCAI'95. Morgan Kaufmann Publishers Inc. San Francisco, USA 1995:1137-43.

13. Shi HY, Lee KT, Lee HH, et al. Comparison of artificial neural network and logistic regression models for predicting in-hospital mortality after primary liver cancer surgery. PloS One. 2012; 7: 1-6.

14. Biglarian A, Bakhshi E, Gohari MR, et al. Artificial neural network for prediction of distant metastasis in colorectal cancer. Asian Pac J Cancer Prev. 2012; 13: 927-30.

15. Chien CW, Lee YC, Ma T, et al. The application of artificial neural networks and decision tree model in predicting post-operative complication for gastric cancer patients. Hepatogastroenterology. 2008; 55: 1140-5.

16. Barlin JN, Zhou Q, Clair CM, et al. Classification and regression tree (CART) analysis of endometrial carcinoma: Seeing the forest for the trees. Gynecol Oncol. 2013; 130: 452-6.

17. Surucu M, Shah KK, Mescioglu I, et al. Decision trees predicting tumor shrinkage for head and neck cancer: implications for adaptive radiation therapy. Technol Cancer Res Treat. 2016; 15: 139-45.

18. Anagnostopoulos I, Anagnostopoulos C, Vergados D, et al. The Wisconsin breast cancer problem: Diagnosis and TTR/DFS time prognosis using probabilistic and generalised regression information classifiers. Oncol Rep. 2006; 15: 975-81.

19. Orr RK. Use of a probabilistic neural network to estimate the risk of mortality after cardiac surgery. Medical Decision Making. 1997; 17: 178-85.

20. Kluska J, Kusy M, Obrzut B. Prediction of radical hysterectomy complications for cervical cancer using computational intelligence methods. In: Rutkowski L, Korytkowski M, Sherer R, Tadeusiewicz R, Zadeh LA, Zurada J, ed. Artificial Intelligence and Soft Computing: 11th International Conference, ICAISC 2012, Zakopane, Poland, April 29-May 3, 2012, Proceedings, Part II. Berlin: Springer-Verlag. 2012; 259-67.

21. Kusy M, Obrzut B, Kluska J. Application of gene expression programming and neural networks to predict adverse events of radical hysterectomy in cervical cancer patients. Med Biol Eng Comput. 2013; 51: 1357-65.

22. Stehman FB, Bundy BN, Disaia PJ, et al. Carcinoma of the cervix treated with radiation therapy I. A multi-variate analysis of prognostic variables in the gynecologic oncology group. Cancer. 1991; 67: 2776-85.

23. Fyles AW, Pintilie M, Kirkbride P, et al. Prognostic factors in patients with cervix cancer treated by radiation therapy: results of a multiple regression analysis. Radiother Oncol. 1995; 35: 107-17.

24. Obrzut B, Semczuk A, Naróg M, et al. Prognostic parameters for patients with cervical cancer FIGO stages IA2-IIB: a long-term follow-up. Oncology. 2017; 93: 106-14.

25. Suprasert P, Srisomboon J, Charoenkwan K, et al. Twelve years experiences with radical hysterectomy and pelvic lymphadenectomy in early stage cervical cancer. J Obstet Gynaecol. 2010; 30: 294-8.

26. Horn LC, Fischer U, Raptis G, et al. Tumor size is of prognostic value in surgically treated FIGO stage II cervical cancer. Gynecol Oncol. 2007; 107: 310-5.

27. Eifel PJ, Jhingran A, Levenback CF, et al. Predictive value of a proposed subclassification of stages I and II cervical cancer based on clinical tumor diameter. Int J Gynecol Cancer. 2009; 19: 2-7.

28. Wagner AE, Pappas L, Ghia AJ, et al. Impact of tumor size on survival in cancer of the cervix and validation of stage IIA1 and IIA2 subdivisions. Gynecol Oncol. 2013; 129: 517-21. 
29. Delgado G, Bundy B, Zaino R, et al. Prospective surgical-pathological study of disease-free interval in patients with stage IB squamous cell carcinoma of the cervix: a Gynecologic Oncology Group study. Gynecol Oncol. 1990; 38: 352-7.

30. Sedlis A, Bundy BN, Rotman MZ, et al. A randomized trial of pelvic radiation therapy versus no further therapy in selected patients with stage IB carcinoma of the cervix after radical hysterectomy and pelvic lymphadenectomy: A Gynecol Oncol. Group Study. Gynecol Oncol. 1999; 73: 177-83.

31. Zreik TG, Chambers JT, Chambers SK. Parametrial involvement, regardless of nodal status: a poor prognostic factor for cervical cancer. Obstet Gynecol. 1996; 87: 741-6.

32. Winer I, Alvarado-Cabrero I, Hassan O, et al. The prognostic significance of histologic type in early stage cervical cancer - A multi-institutional study. Gynecol Oncol. 2015; 137: 474-8.

33. Alfsen GC, Kristensen GB, Skovlund E, et al. Histologic subtype has minor importance for overall survival in patients with adenocarcinoma of the uterine cervix: a population-based study of prognostic factors in 505 patients with non-squamous cell carcinomas of the cervix. Cancer. 2001; 92: 2471-83.

34. Lee KBM, Lee JM, Park CY, et al. What is the difference between squamous cell carcinoma and adenocarcinoma of the cervix? A matched case-control study. Int J Gynecol Cancer. 2006; 16: 1569-73.

35. Takeda N, Sakuragi N, Takeda M, et al. Multivariate analysis of histopathologic prognostic factors for invasive cervical cancer treated with radical hysterectomy and systematic retroperitoneal lymphadenectomy. Acta Obstet Gynecol Scand. 2002; 81: 1144-51.

36. Davy ML, Dodd TJ, Luke CG, et al. Cervical cancer: effect of glandular cell type on prognosis, treatment, and survival. Obstet Gynecol. 2003; 101: 38-45.

37. Landoni F, Maneo A, Cormio G, et al. Class II versus class III radical hysterectomy in stage IB-IIA cervical cancer: a prospective randomized study. Gynecol Oncol. 2001; 80: 3-12.

38. Van Nagell J, Donaldson E, Parker J, et al. The prognostic significance of pelvic lymph node morphology in carcinoma of the uterine cervix. Obstet Gynecol Surv. 1978; 33: 201-4.

39. Zaino R, Ward S, Delgado G, et al. Histopathologic predictors of the behavior of surgically treated stage IB squamous cell carcinoma of the cervix a gynecologic oncology group study. Cancer. 1992; 69: 1750-8.

40. Burghardt E, Pickel H, Haas J, et al. Prognostic factors and operative treatment of stages IB to IIB cervical cancer. Am J Obstet Gynecol. 1987; 156: 988-96.

41. Creasman WT, Zaino RJ, Major FJ, et al. Early invasive carcinoma of the cervix (3 to $5 \mathrm{~mm}$ invasion): risk factors and prognosis: a Gynecol Oncol. Group study. Am J Obstet Gynecol. 1998; 178: 62-5.

42. Inoue T. Prognostic significance of the depth of invasion relating to nodal metastases, parametrial extension, and cell types: A study of 628 cases with stage IB, IIA, and IIB cervical carcinoma. Cancer. 1984; 54: 3035-42.

43. Obrzut B. Impact of the extent of surgery on the treatment outcomes in patients with cervical cancer FIGO IA2-IIB. Poznan: Ośrodek Wydawnictw Naukowych; 2008. [In Polish]

44. Kamura T, Tsukamoto N, Tsuruchi N, et al. Multivariate analysis of the histopathologic prognostic factors of cervical cancer in patients undergoing radical hysterectomy. Cancer. 1992; 69: 181-6.

45. Kristensen GB, Abeler VM, Risberg B, et al. Tumor size, depth of invasion, and grading of the invasive tumor front are the main prognostic factors in early squamous cell cervical carcinoma. Gynecol Oncol. 1999; 74: 245-51.

46. Rutledge FN, Mitchell MF, Munsell M, et al. Youth as a prognostic factor in carcinoma of the cervix: a matched analysis. Gynecol Oncol. 1992; 44: 123-30.

47. Austin J, Degefu S, Torres J, et al. Cervical carcinoma in women less than 35 years of age. South Med J. 1994; 87: 375-9.

48. Stuart G, Robertson D, Fedorkow D, et al. Recurrent and persistent squamous cell cervical carcinoma in women under age 35. Gynecol Oncol. 1988; 30: 163-72.

49. Dattoli MJ, Gretz HF, Beller U, et al. Analysis of multiple prognostic factors in patients with stage IB cervical cancer: Age as a major determinant. Int J Radiat Oncol Biol Phys. 1989; 17: 41-7.

50. Lee YY, Choi CH, Kim TJ, et al. A comparison of pure adenocarcinoma and squamous cell carcinoma of the cervix after radical hysterectomy in stage IB-IIA. Gynecol Oncol. 2011; 120: 439-43.

51. Brewer N, Borman B, Sarfati D, et al. Does comorbidity explain the ethnic inequalities in cervical cancer survival in New Zealand? A retrospective cohort study. BMC Cancer. 2011; 11: 132-8.

52. Burke HB, Goodman PH, Rosen DB, et al. Artificial neural networks improve the accuracy of cancer survival prediction. Cancer. 1997; 79: 857-62.

53. Polterauer S, Grimm C, Hofstetter G, et al. Nomogram prediction for overall survival of patients diagnosed with cervical cancer. Br J Cancer. 2012; 107: 918-24.

54. Ochi T, Murase K, Fujii T, et al. Survival prediction using artificial neural networks in patients with uterine cervical cancer treated by radiation therapy alone. Int J Clin Oncol. 2002; 7: 294-300.

55. Zhou H, Li X, Zhang Y, et al. Establishing a nomogram for stage IA-IIB cervical cancer patients after complete resection. Asian Pacific J Cancer Prev. 2015; 16: 3773-7.

56. [Internet] NCCN: Practice Guidelines in Oncology. Version 1.2019 - August 9, 2018. http://www.nccn.org/
57. Bermudez A, Bhatla N, Leung E. Cancer of the cervix uteri. Int J Gynaecol Obstet. 2015; 131 Suppl. 2: S88-S95.

58. Newton CL, Mould TA. Invasive cervical cancer. Obstetrics, Gynaecology and Reproductive Medicine. 2017; 27: 7-13.

59. Kasamatsu T, Onda T, Sawada M, et al. Radical hysterectomy for FIGO stage IIB cervical cancer: clinicopathological characteristics and prognostic evaluation. Gynecol Oncol. 2009; 114: 69-74.

60. Mabuchi S, Okazawa M, Matsuo K, et al. Impact of histological subtype on survival of patients with surgically-treated stage IA2-IIB cervical cancer: adenocarcinoma versus squamous cell carcinoma. Gynecol Oncol. 2012; 127: $114-20$.

61. Chai Y, Wang T, Wang J, et al. Radical hysterectomy with adjuvant radiotherapy versus radical radiotherapy for FIGO stage IIB cervical cancer. BMC Cancer. 2014; 14: 63-70.

62. Lee YJ, Kim DY, Lee SW, et al. A postoperative scoring system for distant recurrence in node-positive cervical cancer patients after radical hysterectomy and pelvic lymph node dissection with para-aortic lymph node sampling or dissection. Gynecol Oncol. 2017; 144: 536-40.

63. [Internet] DGGG/AGO/DKG. S3-Leitlinie Diagnostik, Therapie und Nachsorge der Patientin mit Zervixkarzinom. Version 1.0 - September 2014. http://www.awmf.org/

64. Kodama J, Seki N, Ojima Y, et al. Prognostic factors in node-positive patients with stage IB-IIB cervical cancer treated by radical hysterectomy and pelvic lymphadenectomy. Int J Gynecol Obstet. 2006; 93: 130-5.

65. Kodama J, Seki N, Ojima Y, et al. Prognostic factors in pathologic parametrium-positive patients with stage IB-IIB cervical cancer treated by radical surgery and adjuvant therapy. Gynecol Oncol. 2007; 105: 757-61. 